\title{
Solar prominence magnetic configurations derived numerically from convection
}

\author{
I. McKaig* \\ Department of Mathematics, Tidewater Community College, Virginia Beach, VA 23456, USA
}

Received 19 January 2001 / Accepted 28 February 2001

\begin{abstract}
The solar convection zone may be a mechanism for generating the magnetic fields in the corona that create and thermally insulate quiescent prominences. This connection is examined here by numerically solving a diffusion equation with convection (below the photosphere) matched to Laplace's equation (modeling the current free corona above the photosphere). The types of fields formed resemble both Kippenhahn-Schlüter and KuperusRaadu configurations with feet that drop into supergranule boundaries.
\end{abstract}

Key words. supergranulation - convection - prominences

\section{Prominence models}

The atmosphere of the Sun, from the base of the chromosphere through the corona, is structured by magnetic fields. Presumably generated in the convection zone these fields break through the photosphere stimulating a variety of plasma formations, from small fibrils that outline convection cells to gigantic solar quiescent prominences that tower above the surface. In this paper we attempt a connection between the ejection of magnetism by convection cells to the structure of these prominences. The term "prominence" can refer to any formation of cool dense material in the corona but a quiescent prominence is the largest and most stable of these formations. First observed during eclipses in the middle ages, they have been described as both "clouds in the lunar atmosphere" and "mountains on the Sun". With the development of photographic and spectrographic methods they are now known to be glowing masses of gas suspended in the Sun's atmosphere by magnetic fields.

The first mathematical models were developed by Menzel (1951) and Dungey (1953). These early models proved unrealistic. For example, Menzel's model predicted prominences that were as wide as they were tall. Better models were developed by Kippenhahn \& Shlüter (KS) (1957) and Kuperus \& Raduu (KR) (1974). These models have better withstood the test of time and are the basis for much work on quiescent prominences. For detailed accounts of the history and physics of prominences see the books "Solar Prominences" by Tandberg-Hanssen (1974) and "Dynamics and Structure of Quiescent Solar Prominences" edited by Priest (1989).

* e-mail: tcmckai@tc.cc.va.us
Marvelous photographs of these magnificent structures can also be found on the internet - see for example http://mesola.obspm.fr (a site run by INSU/CNRS France) and http://sohowww.estec.esa.nl (the web site of the Solar and Heliospheric Observatory).

The field lines in the models by KS and KR are line tied to the photosphere (see McKaig 2001). In a previous paper (McKaig 2001) photospheric motions were simulated by a one-dimensional boundary condition and KS/KR type fields were obtained in the corona. In this paper we will numerically derive magnetic field lines in the corona from two-dimensional convection below the photosphere.

\section{Magnetoconvection}

The basic equation of kinematical magnetoconvection, where a velocity field $\boldsymbol{v}$ is imposed on a plasma and its effect on the magnetic field $\boldsymbol{B}$ computed, is

$\frac{\partial \boldsymbol{B}}{\partial t}=\nabla \times(\boldsymbol{v} \times \boldsymbol{B})+\eta \nabla^{2} \boldsymbol{B}$,

where $\eta$ is the magnetic diffusivity. This equation has been solved both analytically and numerically in various geometries. Some examples are Parker (1963) who obtains exact solutions on infinite and semi-infinite intervals, and Weiss (1977) who numerically solves the equation over finite domains. For a comprehensive review of magnetoconvection see Proctor \& Weiss (1982) and Weiss (1966).

In much of magnetoconvection the fluid fills the whole of the domain and the boundaries are assumed to be perfectly conducting. This ties the magnetic field lines to the boundary and confines the field to the region of fluid flow. Even though the equation is time dependent, an initially 


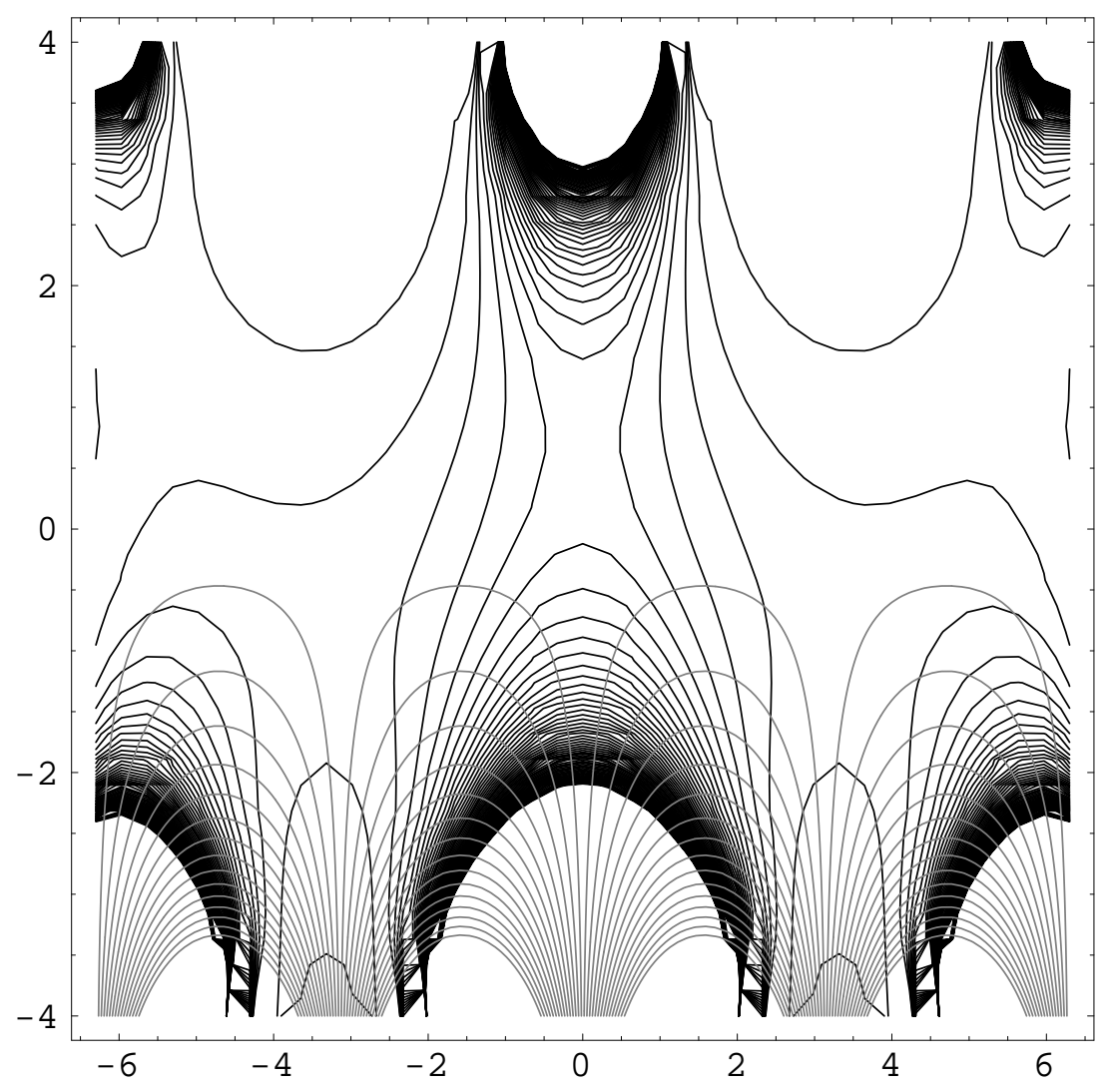

Fig. 1. The stream function $\psi(x, y)=-\sin x \sinh y$ with magnetic field lines computed using the boundary conditions: $g 1(y)=$ $g 2(y)=0, h 1(x)=0, h 2(x)=100$

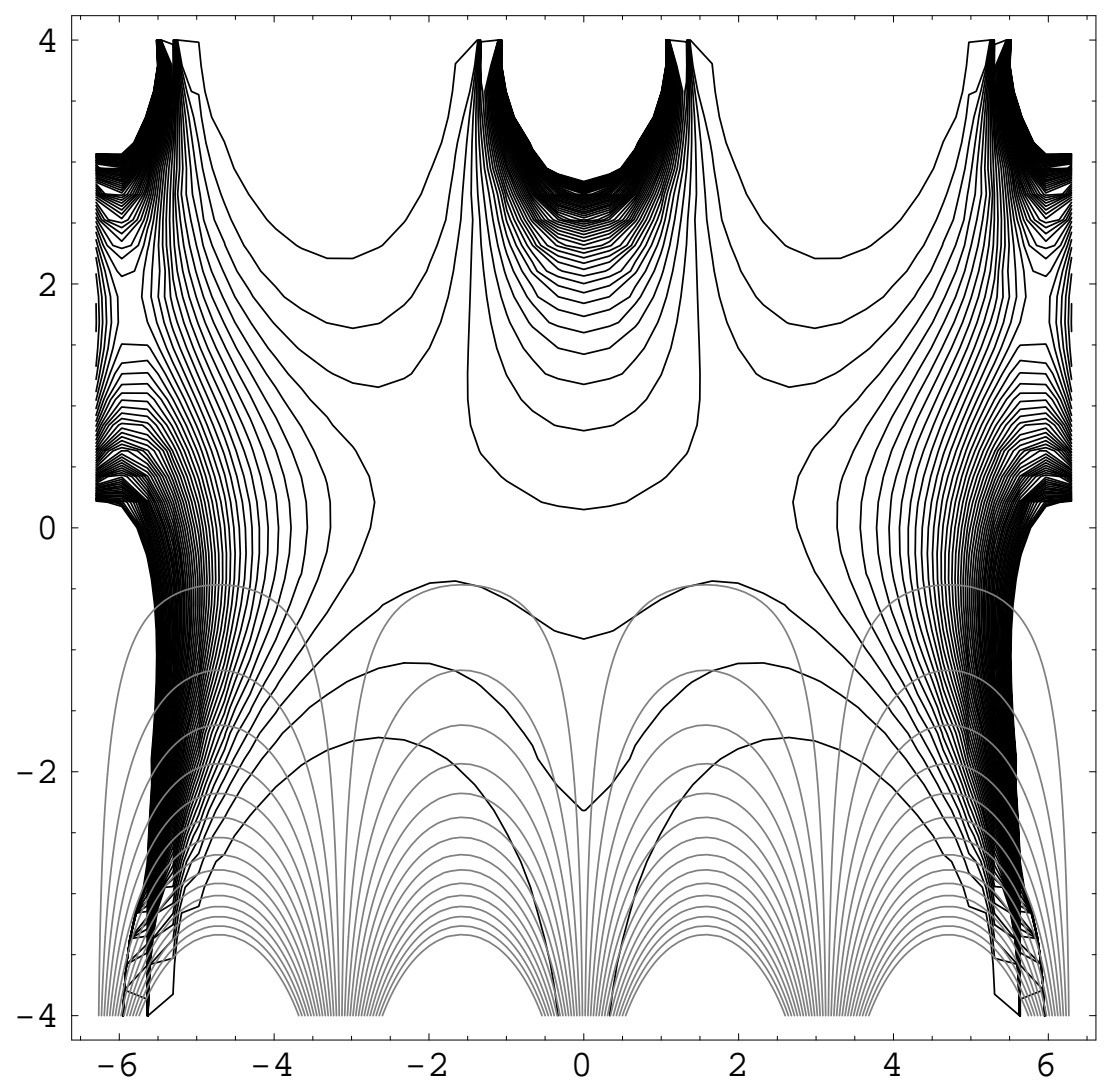

Fig. 2. The stream function $\psi(x, y)=-\sin x \sinh y$ with magnetic field lines computed using the boundary conditions: $g 1(y)=$ $g 2(y)=\left\{\begin{array}{ll}100, & y<0 \\ 0, & y>0\end{array}, h 1(x)=h 2(x)=0\right.$ 


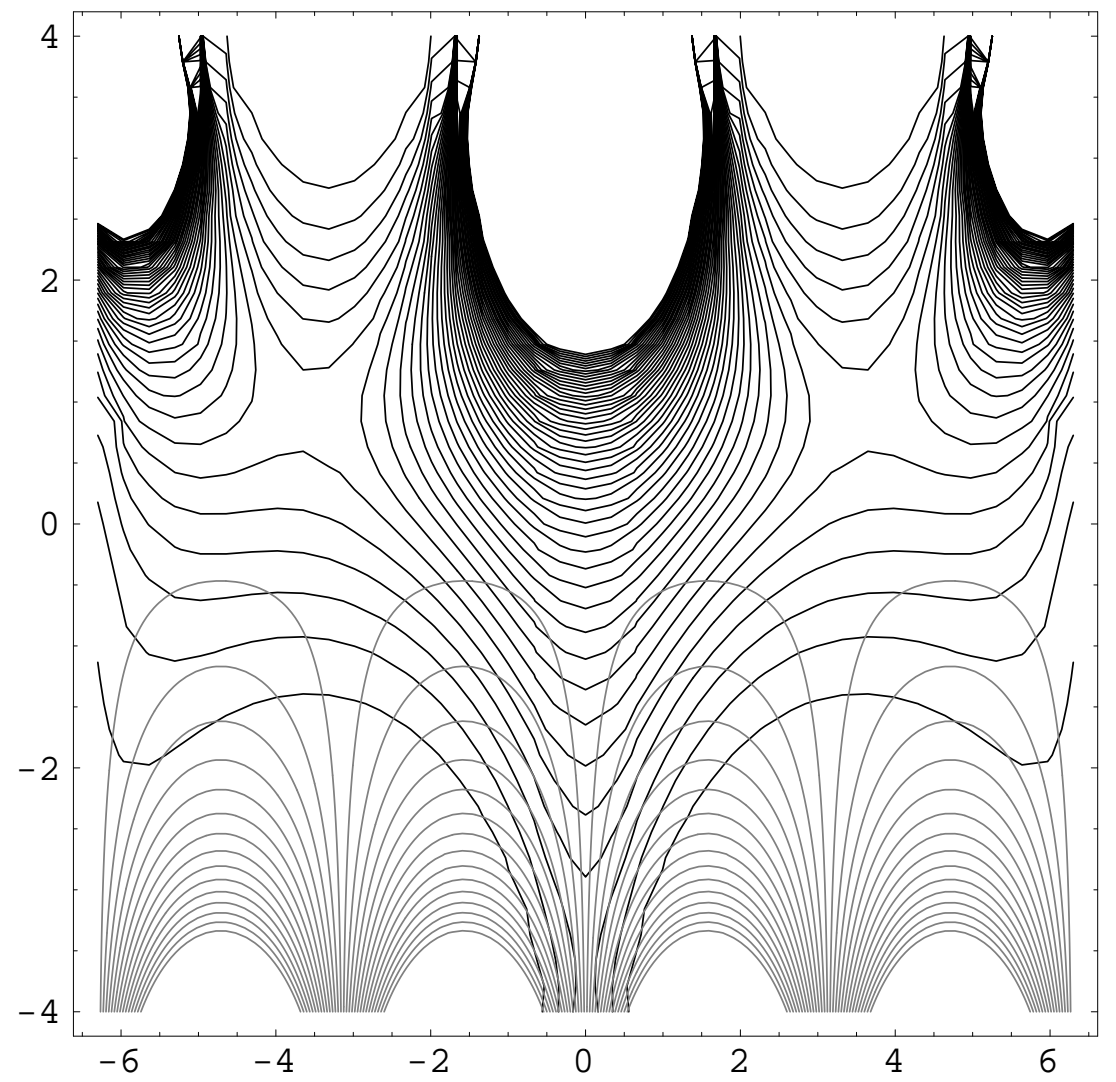

Fig. 3. The stream function $\psi(x, y)=-\sin x \sinh y$ with magnetic field lines computed using the boundary conditions: $g 1(y)=$ $g 2(y)=0, h 1(x)=100, h 2(x)=0$

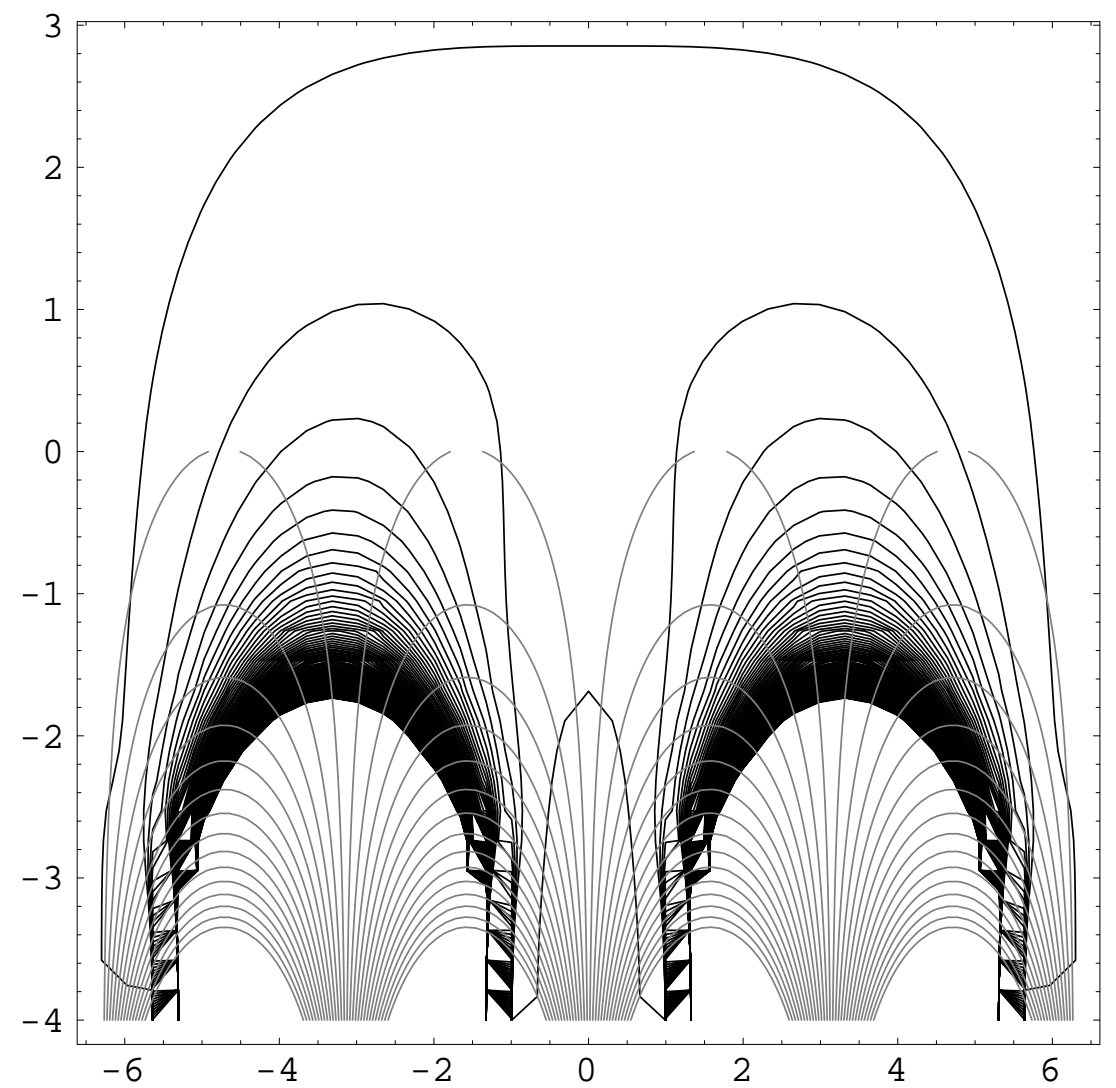

Fig. 4. The stream function $\psi(x, y)=-\sin (x) e^{-y}$ with magnetic field lines computed using the boundary conditions: $g 1(y)=$ $g 2(y)=0, h 1(x)=0, h 2(x)=100$ 


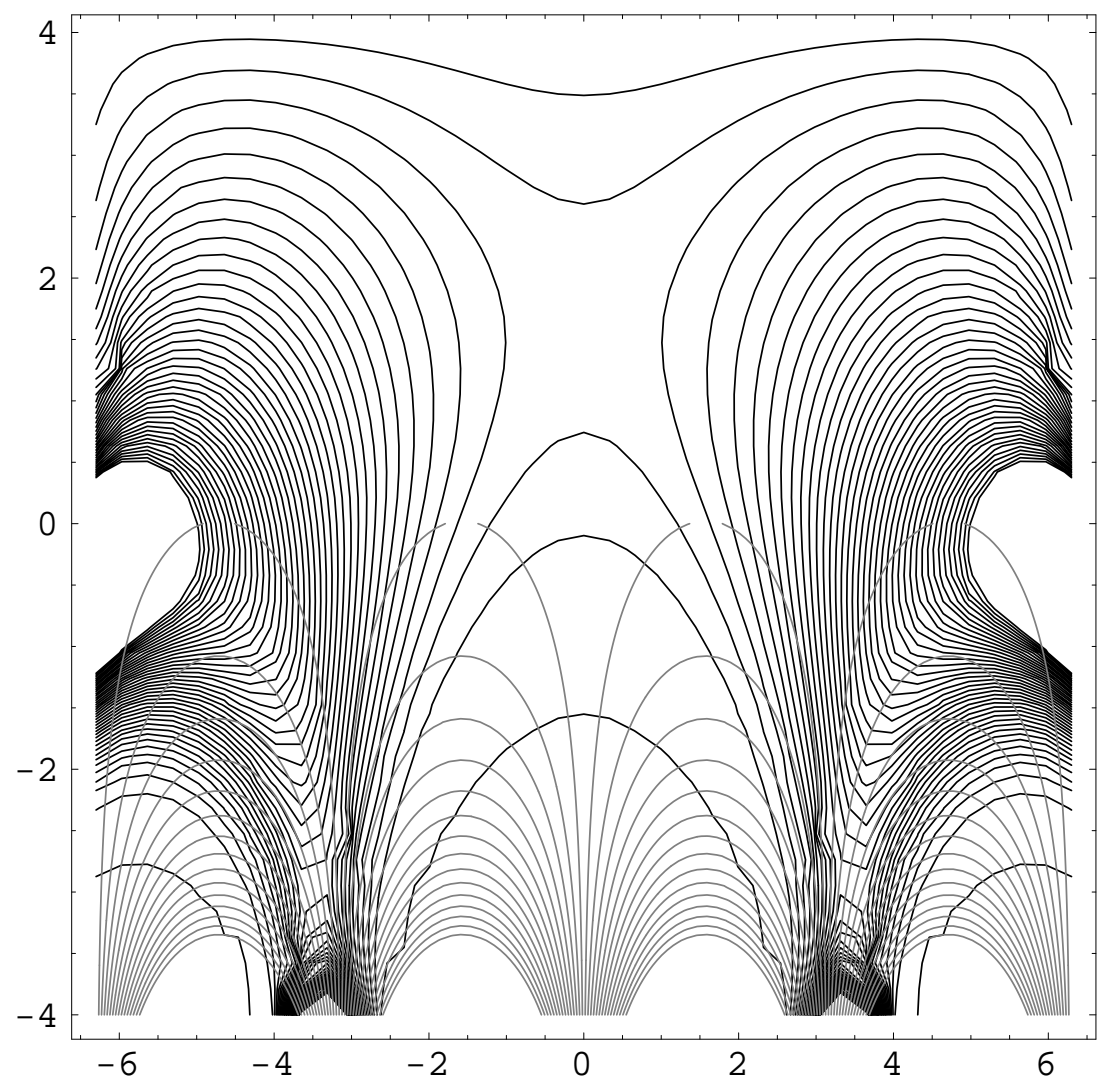

Fig. 5. The stream function $\psi(x, y)=-\sin (x) e^{-y}$ with magnetic field lines computed using the boundary conditions: $g 1(y)=$ $g 2(y)=\left\{\begin{array}{ll}100, & y<0 \\ 0, & y>0\end{array}, h 1(x)=h 2(x)=0\right.$

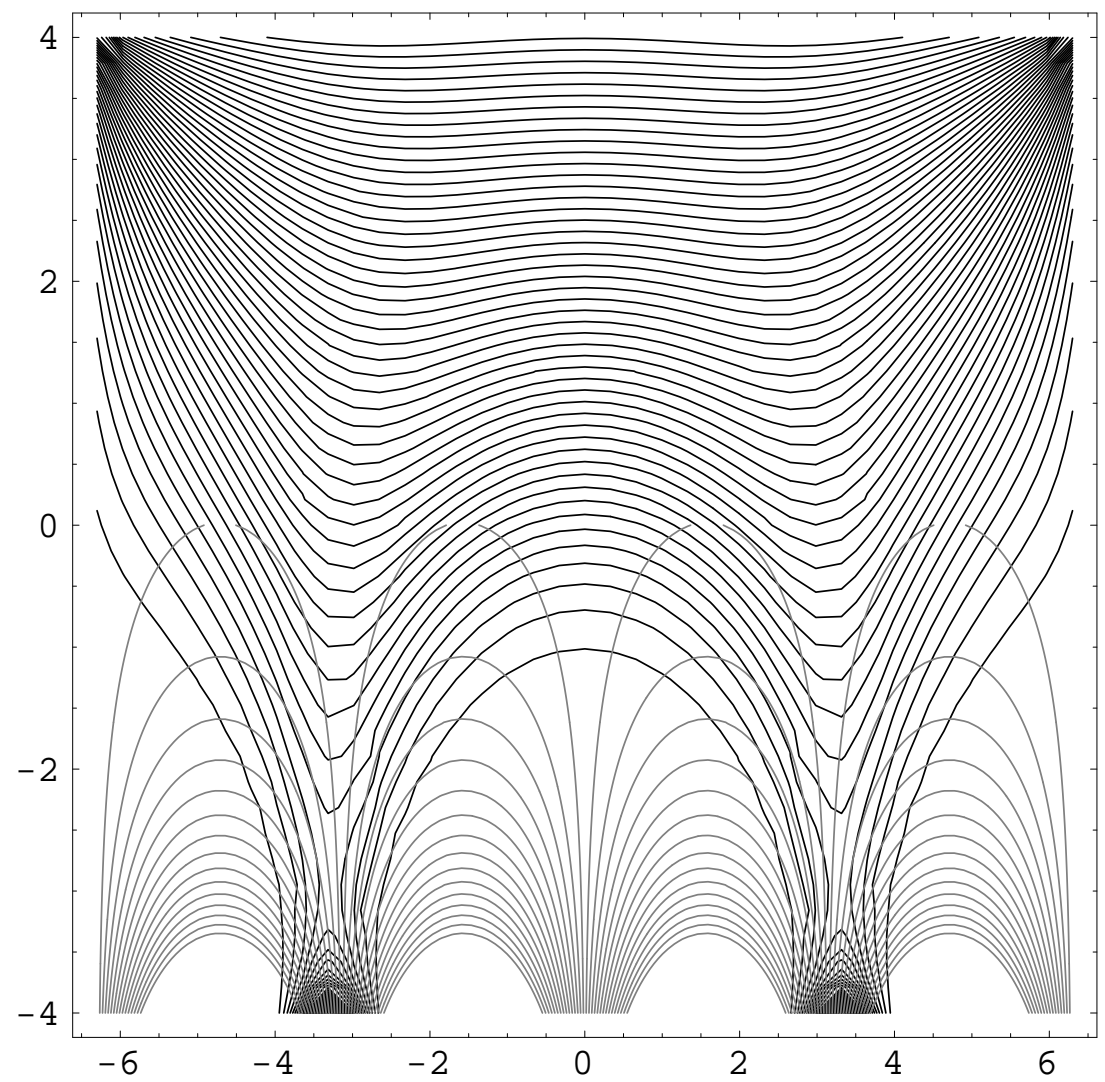

Fig. 6. The stream function $\psi(x, y)=-\sin (x) e^{-y}$ with magnetic field lines computed using the boundary conditions: $g 1(y)=$ $g 2(y)=0, h 1(x)=100, h 2(x)=0$ 
uniform field is expelled by the convection to the boundaries and reaches a steady-state. In an attempt to have convection expel magnetism from below the photosphere into the corona we will solve (1) with $\frac{\partial}{\partial t} \equiv 0$ (steady state has been achieved) on the interval $-L<x<L$ and $-d<y<d$. The convection zone will be $y<0$ where $\boldsymbol{v}$ will be prescribed, while $y>0$ will be a current free corona where $\nabla \times \boldsymbol{B}=\mathbf{0}$.

\section{The mathematical model}

If a vector potential $A(x, y)$ is introduced such that $\boldsymbol{B}=$ $\nabla \times(0,0, A)=\left(\frac{\partial A}{\partial y},-\frac{\partial A}{\partial x}, 0\right)$, then the contours given by $A=$ constant will give the magnetic field lines, $A$ being essentially a stream function for $\boldsymbol{B}$. In the corona $(y>0)$, $\nabla \times \boldsymbol{B}=\mathbf{0}$ becomes Laplace's equation, $\nabla^{2} A=0$. In the convection zone $(y<0)$, we cast $(1)$ with $\frac{\partial}{\partial t} \equiv 0$ in $2-\mathrm{D}$ which in terms of $A$ gives a diffusion equation with convection:

$v_{1}(x, y) \frac{\partial A}{\partial x}+v_{2}(x, y) \frac{\partial A}{\partial y}-\eta \nabla^{2} A=0$,

where $\boldsymbol{v}=\left\langle v_{1}, v_{2}\right\rangle$ is known. This equation can be changed to a Helmholtz equation by the transformation $A=\Lambda(x, y) e^{H(x, y)}$ where $H=\frac{\phi}{2 \eta}$ and $\phi(x, y)$ is the velocity potential. This gives

$\eta \nabla^{2} \Lambda+f(x, y) \Lambda=0$

where

$f(x, y)=\frac{1}{4}\left[2\left(\frac{\partial v_{1}}{\partial x}+\frac{\partial v_{2}}{\partial y}\right)-\frac{1}{\eta}\left(v_{1}^{2}+v_{2}^{2}\right)\right]$.

To make this transformation possible $\boldsymbol{v}$ is restricted to being conservative but it makes the numerical solution more tractable. It not only eliminates the first derivative terms but since $\boldsymbol{v} \equiv 0$ in the corona $\nabla^{2} A=0$ becomes $\nabla^{2} \Lambda=0$ under the transformation $A=\Lambda e^{H}$. So by setting $f(x, y)=0$ for $y>0$ we force the two solutions to match at the photosphere.

\section{The numerical method}

The numerical method used was the finite difference method. Equation (3) was solved on the computational box $-L<x<L$ and $-d<y<d$, with $f(x, y)$ set to zero for $0<y<d$. The box was split into an $n \times m$ mesh and the derivatives were approximated by a central difference scheme:

$u_{x x} \approx \frac{u\left(x_{i+1}, y_{j}\right)-2 u\left(x_{i}, y_{j}\right)+u\left(x_{i-1}, y_{j}\right)}{h^{2}}$

$u_{y y} \approx \frac{u\left(x_{i}, y_{j+1}\right)-2 u\left(x_{i}, y_{j}\right)+u\left(x_{i}, y_{j-1}\right)}{k^{2}}$

where $h=\frac{2 L}{n}$ and $k=\frac{2 d}{m}$. The boundary conditions were specified as $g 1(y)$ and $g 2(y)$ on $x=L$ and $x=-L$ respectively and $h 1(x)$ and $h 2(x)$ on $y=d$ and $y=-d$ respectively. The scheme was programmed on Mathematica which has well tested subroutines for solving the large linear systems created by the finite difference method. Once the solution for $\Lambda(x, y)$ was obtained it was multiplied by $\mathrm{e}^{\frac{\phi}{2 \eta}}$ to get the solution for $A(x, y)$. A contour plot of $A$ then gives the magnetic field lines.

\section{Results}

All runs were made with $L=6.3 \approx 2 \pi$ and $d=4$. The first stream function used was $\psi(x, y)=-\sin x \sinh y$. This models a deep convection zone with a downflow at the center. This stream function was used with different boundary conditions in Figs. 1, 2 and 3. The stream function has been overlayed with the field lines.

The next stream function, $\psi(x, y)=-\sin (x) e^{-y}$, has an upflow at the center and the same boundary conditions were applied in Figs. 4, 5 and 6. An exact solution to (1) with $\frac{\partial}{\partial t} \equiv 0$ using this stream function was obtained by Parker (1963) for $x, y \rightarrow \pm \infty$. Figure 6 resembles his solution.

\section{Conclusions}

This model shows that convection is capable of expelling magnetic fields into the corona which have the topology needed to form quiescent prominences. Many of the diagrams have "feet" that form in the downflow regions of the convection cells. The fields have the magnetic dips present in the $\mathrm{KS}$ model as well as the sheet type formations of the KR model. This provides many regions where plasma can condense thus accounting for the filamentry structure observed in quiescent prominences.

Acknowledgements. This paper was written under the auspices of the Department of Mathematics and Statistics, Old Dominion University, Norfolk VA. I am grateful to Dr. John Adam, my Ph.D. advisor and the referee for their suggestions on improving this article.

\section{References}

Dungey, J. W. 1953, MNRAS, 113, 180

Kippenhahn, R., \& Schlüter, A. 1957, Z. Astrophys., 43, 36

Kuperus, M., \& Raadu, M. A. 1974, A\&A, 123, 80

McKaig, I. 2001, A\&A, 368, 280

Menzel, D. H. 1951, Proceedings of the Conference on Dynamics of Ionized Media at University College, London Parker, E. N. 1963, ApJ, 138, 552

Priest, E. R. 1989, Dynamics and Structure of Quiescent Solar Prominences, ed. E. R. Priest (Kluwer Academic Publishers, Dordrecht, Holland)

Proctor, M. R. E., \& Weiss, N. O. 1982, Rep. Prog. Phys., 45, 1317

Tandberg-Hanssen, E. 1974, Solar Prominences (D. Reidel Publ. Co., Dordrecht, Holland)

Weiss, N. O. 1966, Proc. Roy. Soc. Lond. A., 293, 310

Weiss, N. O. 1977, Problems of Stellar Convection, ed. E. A. Spiegel, \& J. P. Zahn (Springer-Verlag, Berlin), 176 